\title{
Distributed Control Applied to Combined Electricity and Natural Gas Infrastructures
}

\author{
Michèle Arnold, Rudy R. Negenborn, Göran Andersson, Bart De Schutter
}

\begin{abstract}
The optimization of combined electricity and natural gas systems is addressed in this paper. The two networks are connected via energy hubs. Using the energy hub concept, the interactions between the different infrastructures can be analyzed. A system consisting of several interconnected hubs forms a distributed power generation structure where each hub is controlled by its respective control agent. Recently, a distributed control method has been applied to such a system. The overall optimization problem including the entire system is decomposed into subproblems according to the control agents. In this paper, a parallel and serial version of that method is discussed. Simulation results are obtained through experiments on a three-hub benchmark system.
\end{abstract}

\section{INTRODUCTION}

$\mathbf{M}$ ULTI-carrier networks are power delivery systems that are not restricted to a single energy carrier, such as electricity. Instead, they consider multiple energy carriers, such as natural gas, hydrogen, or local district heating systems. Recently, an integrated view of these various energy systems has been suggested [1], [2], [3] mainly due to the increased utilization of gas-fired and other distributed generation, especially co- and trigeneration [3]. The various available energy carriers and the possible conversion between them significantly affect both the technical and the economical operation of energy systems. In particular, consumers get flexibility in supply and can therefore decide depending on criteria such as cost, reliability, system emissions, or availability. The couplings and interactions between the different energy carriers are covered with the concept of "energy hubs" [2]. Energy systems are then considered as consisting of a number of interconnected energy hubs, which together form a distributed power generation structure.

In this paper an optimal power flow problem for an integrated electricity and natural gas system is studied. In [2], a centralized control scheme has been applied to such a system, in which one optimization problem including the entire system is solved by a single central control agent. In [4], the optimal power flow problem has been solved in a distributed way, in which each hub is controlled by its respective control agent. The decomposition method presented in [5] is applied there, dividing the overall optimization

M. Arnold and G. Andersson are with the Power Systems Laboratory, ETH Zurich, Physikstrasse 3, 8092 Zurich, Switzerland, email: $\{$ arnold, andersson $\} @ e e h$. ee. ethz.ch. R.R. Negenborn and B. De Schutter are with the Delft Center for Systems and Control of the Delft University of Technology, Mekelweg 2, 2628 CD Delft, The Netherlands, e-mail: r.r.negenborn@tudelft.nl, b.deschutter@dcsc.tudelft.nl. B. De Schutter is also with the Marine and Transport Technology department of the Delft University of Technology. problem into subproblems, according to the control agents. These subproblems are solved within an iterative procedure, separately, but coordinated. In order to guarantee the energy supply of the entire system, the control agents have to coordinate their actions among one another. A distributed control procedure is advantageous as it better suits a distributed power generation infrastructure. Moreover, less data transfer and higher robustness are provided, in particular for largescale systems.

In this paper, we focus on the communication among the control agents. To achieve coordination, the control agents exchange dedicated information among one another. This information exchange can take place in various ways [6]. In this paper, we are in particular interested in the differences in performance between serial and parallel schemes [7].

The remainder of this paper is organized as follows. In Section II we introduce the mathematical model used to represent the combined electricity and natural gas network. In Section III the control objectives from a network-wide point of view and from a single-area point of view are formulated. In Section III we also discuss two implementations of a particular distributed control scheme: a serial and a parallel implementation. In Section IV simulations are carried out on a three-hub benchmark system to compare the serial and parallel scheme. Section V concludes this paper and outlines directions for future research.

\section{MODELING}

The combined electricity and natural gas network we consider in this paper is described below. The equations for power flow within energy hubs (energy conversion) and between the hubs (energy transmission) are given. We consider a model that captures the static steady-state characteristics of the network, as it is commonly done in optimal power flow studies.

\section{A. System setup}

The considered system consists of three interconnected hubs, as depicted in Fig. 1. The electricity network consists of three electricity buses connected by three transmission lines. Each of the buses has a generator connected to it $\left(\mathrm{G}_{i}\right.$, with electric power production $P_{\mathrm{e}, i}^{\mathrm{G}}$, for $\left.i \in\{1,2,3\}\right)$ and supplies electricity to the three hubs $\left(\mathrm{H}_{i}\right.$, with electricity hub inputs $P_{\mathrm{e}, i}^{\mathrm{H}}$, for $\left.i \in\{1,2,3\}\right)$. The gas network consists of three gas buses. However, only one of these buses is connected to a large gas network, which is considered as gas generator $\mathrm{N}$ with gas production $P_{\mathrm{g}}^{\mathrm{G}}$. Besides this, each of the gas buses provides gas to the three hubs $\left(\mathrm{H}_{i}\right.$, with gas 


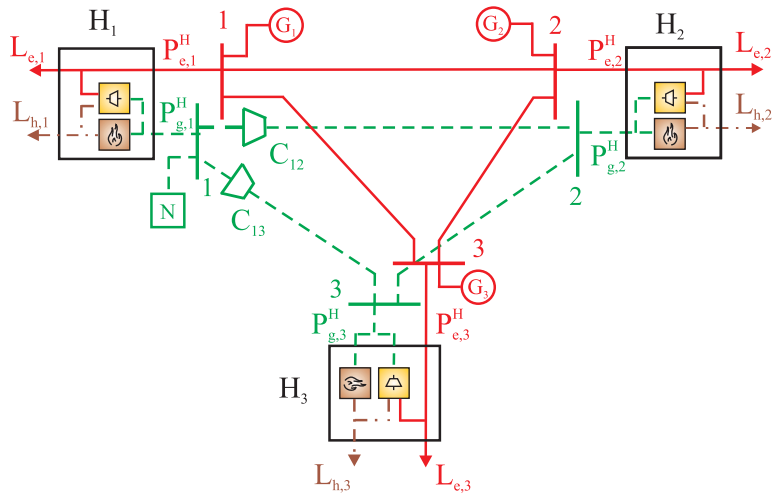

Fig. 1. System setup of three interconnected energy hubs. Active power is provided by generators $\mathrm{G}_{1}, \mathrm{G}_{2}, \mathrm{G}_{3}$ and natural gas is demanded from an adjacent network, modeled as gas generator $\mathrm{N}$.

hub inputs $P_{\mathrm{g}, i}^{\mathrm{H}}$, for $\left.i \in\{1,2,3\}\right)$. Compressors are present within the pipelines that connect the gas buses $\left(\mathrm{C}_{i j}\right.$, for $(i, j) \in\{(1,2),(1,3)\})$. The compressors provide a certain pressure level and thus enable the gas flow to the surrounding gas sinks.

The electricity and gas network are connected via energy hubs. In fact, the energy hub is a generalization and extension of a network node, including conversion, conditioning, and storage of multiple energy carriers. It represents the interface between the energy sources and transmission lines on the one hand and the power consumers on the other hand. Basically, the concept of energy hubs is not restricted to any size of the modeled system. Single power plants or industrial buildings as well as bounded geographical areas such as whole towns and cities can be modeled as energy hubs.

In the system under consideration, each energy hub $i$ takes electric power $P_{\mathrm{e}, i}^{\mathrm{H}}$ and gas $P_{\mathrm{g}, i}^{\mathrm{H}}$ from the electricity and gas network, respectively, and supplies its electric load $L_{\mathrm{e}, i}$ and heat load $L_{\mathrm{h}, i}$. To meet the load requirements at the output, the input energies are appropriately converted within the hub. For the internal energy conversion, a gas turbine and a furnace are included in each of the hubs. The gas turbine couples the two energy systems as it simultaneously produces electricity and heat from natural gas. The electric load $L_{\mathrm{e}, i}$ can be supplied with electric power either directly from the electricity network, or indirectly by converting gas from the gas network using the gas turbine. Due to this redundant path within the energy supply, the reliability of supply is increased. Furthermore, due to this redundancy, the supply energies at the input can be optimized according to criteria such as cost, availability, emissions, etc.

\section{B. Energy hub}

Within an energy hub $i$, power can be converted from one energy carrier $\alpha$ into an energy carrier $\beta$. Considering a single-input single-output converter device, the steady-state input power $P_{\alpha, i}$ and output power $L_{\beta, i}$ are coupled as

$$
L_{\beta, i}=c_{\alpha \beta, i} P_{\alpha, i},
$$

where $c_{\alpha \beta, i}$ characterizes the coupling factor between the input and output power. In this case, the coupling factor corresponds to the converter's steady-state energy efficiency, denoted by $\eta_{\alpha \beta, i}$. Unidirectional power flows within the converters are assumed, i.e., $P_{\alpha, i} \geq 0, P_{\beta, i} \geq 0$. Regarding the model of the entire hub, various converter elements are included, which leads to the following relation:

$$
\underbrace{\left[\begin{array}{c}
L_{\alpha, i} \\
L_{\beta, i} \\
\vdots \\
L_{\omega, i}
\end{array}\right]}_{\mathbf{L}_{i}}=\underbrace{\left[\begin{array}{cccc}
c_{\alpha \alpha, i} & c_{\beta \alpha, i} & \cdots & c_{\omega \alpha, i} \\
c_{\alpha \beta, i} & c_{\beta \beta, i} & \cdots & c_{\omega \beta, i} \\
\vdots & \vdots & \ddots & \vdots \\
c_{\alpha \omega, i} & c_{\beta \omega, i} & \cdots & c_{\omega \omega, i}
\end{array}\right]}_{\mathbf{C}_{i}} \underbrace{\left[\begin{array}{c}
P_{\alpha, i} \\
P_{\beta, i} \\
\vdots \\
P_{\omega, i}
\end{array}\right]}_{\mathbf{P}_{i}},
$$

which expresses how the input powers $P_{\alpha, i}, P_{\beta, i}, \ldots, P_{\omega, i}$ are converted into the output powers $L_{\alpha, i}, L_{\beta, i}, \ldots, L_{\omega, i}$. Matrix $\mathbf{C}_{i}$ is referred to as a coupling matrix.

When considering multiple inputs and outputs, the coupling factors $c_{\alpha \beta, i}$ may be different from the converter efficiencies $\eta_{\alpha \beta, i}$. Energy carriers may be split up into several converters as it is the case for the gas input power $P_{\mathrm{g}, i}^{\mathrm{H}}$ in the considered system setup. In this case, so-called dispatch factors for optimally dispatching the total input power to the correspondent converter devices have to be incorporated in the coupling factors. As indicated in Fig. 1, the gas input $P_{\mathrm{g}, i}^{\mathrm{H}}$ is split up into two parts. The part $\nu_{\mathrm{g}, i} P_{\mathrm{g}, i}^{\mathrm{H}}$ defines the gas input power fed into the gas turbine and the part $\left(1-\nu_{\mathrm{g}, i}\right) P_{\mathrm{g}, i}^{\mathrm{H}}$ defines the gas input power going into the furnace. The dispatch factor $\nu_{\mathrm{g}, i}\left(0 \leq \nu_{\mathrm{g}, i} \leq 1\right)$ defines the ratio. Finally, the coupling factors $c_{\alpha \beta, i}$ for converters without explicitly preassigned inputs are defined as the product of dispatch factor and converter efficiency, i.e., $c_{\alpha \beta, i}=\nu_{\mathrm{g}, i} \eta_{\alpha \beta, i}$.

For the energy hub depicted in Fig. 1, the conversion from the electric input power $P_{\mathrm{e}, i}^{\mathrm{H}}$ and the gas input power $P_{\mathrm{g}, i}^{\mathrm{H}}$ into the electric output power $L_{\mathrm{e}, i}$ and heat output power $L_{\mathrm{h}, i}$ is described by:

$$
\underbrace{\left[\begin{array}{c}
L_{\mathrm{e}, i} \\
L_{\mathrm{h}, i}
\end{array}\right]}_{\mathbf{L}_{i}}=\underbrace{\left[\begin{array}{cc}
1 & \nu_{\mathrm{g}, i} \eta_{\mathrm{ge}, i}^{\mathrm{T}} \\
0 & \nu_{\mathrm{g}, i} \eta_{\mathrm{gh}, i}^{\mathrm{T}}+\left(1-\nu_{\mathrm{g}, i}\right) \eta_{\mathrm{gh}, i}^{\mathrm{F}}
\end{array}\right]}_{\mathbf{C}_{i}} \underbrace{\left[\begin{array}{c}
P_{\mathrm{e}, i}^{\mathrm{H}} \\
P_{\mathrm{g}, i}^{\mathrm{H}}
\end{array}\right]}_{\mathbf{P}_{i}},
$$

where $\eta_{\mathrm{ge}, i}^{\mathrm{T}}$ and $\eta_{\mathrm{gh}, i}^{\mathrm{T}}$ denote the converter electric and thermal efficiencies for the gas turbine $\mathrm{T}$, and $\eta_{\mathrm{gh}, i}^{\mathrm{F}}$ denotes the converter efficiency for the furnace $\mathrm{F}$.

As the dispatch factor $\nu_{\mathrm{g}, i}$ is variable, different power input vectors $\mathbf{P}_{i}$ can be found to fulfill the load requirements $\mathbf{L}_{i}$ at the output. This illustrates the degrees of freedom in supply.

\section{Power transmission networks}

For the transmission networks of both the electricity system and the gas pipeline system, power flow models based on nodal power balances are implemented. The power flows for the electricity network are formulated as nodal power balances of the complex power, according to [4], [8]. The power flow equations for the pipeline network are described in more detail as they are less common. Fig. 2 shows the model of a gas pipeline composed of a compressor and a 


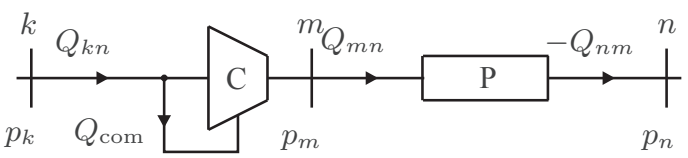

Fig. 2. Model of a gas pipeline with compressor (C) and pipeline (P). Compressor demand is modeled as additional power flow $Q_{\text {com }}$.

pipeline element. The volume flow balance at node $m$ is defined as

$$
Q_{m}-\sum_{n \in \mathcal{N}_{m}} Q_{m n}=0
$$

where $Q_{m}$ is the volume flow injected at node $m, Q_{m n}$ denotes the line flow between nodes $m$ and $n$, and $\mathcal{N}_{m}$ denotes the set of neighboring nodes of node $m$, i.e., the nodes connected to node $m$ through a pipe line. The line flow $Q_{m n}$ is defined as

$$
Q_{m n}=k_{m n} s_{m n} \sqrt{s_{m n}\left(p_{m}^{2}-p_{n}^{2}\right)},
$$

where $p_{m}$ and $p_{n}$ denote the upstream and downstream pressures, respectively, and $k_{m n}$ identifies the line constant. The variable $s_{m n}$ indicates the direction of the gas flow as

$$
s_{m n}= \begin{cases}+1 & \text { if } p_{m} \geq p_{n} \\ -1 & \text { otherwise. }\end{cases}
$$

To maintain a certain pressure level a compressor is needed. Here, the compressor is driven by a gas turbine, which is modeled as additional gas flow

$$
Q_{\text {com }}=k_{\text {com }} Q_{m n}\left(p_{m}-p_{k}\right),
$$

where $p_{k}$ denotes the pressure at the compressor input side and $k_{\mathrm{com}}$ is a compressor constant. The pressure at the compressor output $p_{m}$ is determined by

$$
p_{m}=p_{\text {inc }} p_{k},
$$

where $p_{\text {inc }}$ defines the pressure amplification of the compressor. Depending on the required line flow $Q_{m n}, p_{\text {inc }}$ is adjusted accordingly. For the purpose of this study, these simplified compressor models provide sufficient accuracy. More advanced compressor equations taking into account changing fluid properties are given in [9]. Additional details of the system under study can be found in [2], [4].

\section{Combined electricity and natural gas system}

The combined electricity and gas network is obtained by combining the above stated power flow models. The states $\mathbf{x}$ include the system variables of the electricity and the gas system as well as the system variables of the three hubs:

$$
\mathbf{x}=\left[\begin{array}{llllll}
\mathbf{V} & \boldsymbol{\theta} & \mathbf{p} & \mathbf{p}_{\text {inc }} & \mathbf{P}_{\mathrm{e}}^{\mathrm{H}} & \mathbf{P}_{\mathrm{g}}^{\mathrm{H}}
\end{array}\right]^{\mathrm{T}},
$$

where $\mathbf{V}=\left[V_{1}, V_{2}, V_{3}\right]^{\mathrm{T}}$ and $\boldsymbol{\theta}=\left[\theta_{1}, \theta_{2}, \theta_{3}\right]^{\mathrm{T}}$ denote the voltage magnitudes and angles of the electric buses, $\mathbf{p}=\left[p_{1}, p_{2}, p_{3}\right]^{\mathrm{T}}$ describes the nodal pressures of all gas buses, $\mathbf{p}_{\text {inc }}=\left[p_{\text {inc }, 1}, p_{\text {inc }, 2}\right]^{\mathrm{T}}$ indicates the compressor variables of the two compressors, $\mathbf{P}_{\mathrm{e}}^{\mathrm{H}}=\left[P_{\mathrm{e}, 1}^{\mathrm{H}}, P_{\mathrm{e}, 2}^{\mathrm{H}}, P_{\mathrm{e}, 3}^{\mathrm{H}}\right]^{\mathrm{T}}$ refers to the electric input powers of the hubs and $\mathbf{P}_{\mathrm{g}}^{\mathrm{H}}=$ $\left[P_{\mathrm{g}, 1}^{\mathrm{H}}, P_{\mathrm{g}, 2}^{\mathrm{H}}, P_{\mathrm{g}, 3}^{\mathrm{H}}\right]^{\mathrm{T}}$ to the gas input powers of the hubs.

The control variables $\mathbf{u}$ include the active power generation of all generators, the natural gas import and the dispatch factors of each hub, i.e.,

$$
\mathbf{u}=\left[\begin{array}{lll}
\mathbf{P}_{\mathrm{e}}^{\mathrm{G}} & P_{\mathrm{g}}^{\mathrm{G}} & \boldsymbol{\nu}_{\mathrm{g}}
\end{array}\right]^{\mathrm{T}},
$$

where $\mathbf{P}_{\mathrm{e}}^{\mathrm{G}}=\left[P_{\mathrm{e}, 1}^{\mathrm{G}}, P_{\mathrm{e}, 2}^{\mathrm{G}}, P_{\mathrm{e}, 3}^{\mathrm{G}}\right]^{\mathrm{T}}$ denotes the active power generation of all generators and $\nu_{\mathrm{g}}=\left[\nu_{\mathrm{g}, 1}, \nu_{\mathrm{g}, 2}, \nu_{\mathrm{g}, 3}\right]^{\mathrm{T}}$ describes the dispatch factors of the gas input junctions.

Now, the model we use to represent the combined electricity and gas network can be conveniently written as

$$
\mathrm{g}(\mathbf{x}, \mathbf{u})=\mathbf{0},
$$

summarizing the power flow equations of the electricity and gas system and the hub equations.

\section{CONTROL PROBLEM FORMULATION}

The control variables $\mathbf{u}$ should be set in such a way that the following control objectives are achieved:

- The costs for electricity generation should be minimized.

- The costs for natural gas usage should be minimized.

The control problem can be stated as determining the optimal operational set points $\mathbf{u}$ in such a way that the control objectives are achieved, while satisfying the system constraints $^{1}$ (secondary layer control). Below, we first formulate the overall optimization problem, which is solved within a centralized control scheme. After this, we divide the optimal power flow problem over control agents, which solve their problems in a distributed way.

\section{A. Centralized formulation}

In the centralized control formulation there is a single control agent that determines the inputs for the whole network. The control objectives are adequately represented by the following system-wide objective function:

$$
J(\mathbf{x}, \mathbf{u})=\sum_{i=1}^{n_{G}} q_{i}^{\mathrm{G}}\left(P_{\mathrm{e}, i}^{\mathrm{G}}\right)^{2}+q^{\mathrm{N}}\left(P_{\mathrm{g}}^{\mathrm{G}}\right)^{2},
$$

where $n_{G}$ denotes the number of generators and $q_{i}^{\mathrm{G}}, q^{\mathrm{N}}$ are the quadratic costs on electricity generation and natural gas consumption, respectively. The centralized control problem formulation is now stated as

$$
\min _{\mathbf{u}} J(\mathbf{x}, \mathbf{u})
$$

subject to

$$
\begin{aligned}
& \mathrm{g}(\mathbf{x}, \mathbf{u})=\mathbf{0} \\
& \mathbf{h}(\mathbf{x}, \mathbf{u}) \leq \mathbf{0},
\end{aligned}
$$

where the inequality constraints (15) comprise limits on voltage magnitudes, active and reactive power flows, pressures, changes in compressor settings, and dispatch factors.

\footnotetext{
${ }^{1}$ In addition to the stated objectives, it would be straightforward to also include voltage regulation and power flow limitations as control objectives.
} 
Furthermore, power limitations on hub inputs and on gas and electricity generation are also incorporated in (15).

The optimization problem (13)-(15) is a nonlinear programming problem [10], which can be solved using optimization problem solvers for nonlinear programming, such as sequential quadratic programming [10]. In general, the solution space is non-convex and therefore finding the global optimum cannot be guaranteed.

\section{B. Distributed formulation}

In the distributed control formulation the assumption is made that instead of having a single control agent responsible for the operation of the entire network, there are three control agents, each of them responsible for a particular part of the network. Each control agent is responsible for the hub variables and all system variables of the nodes connected to it. Hence, control area 1 comprises the hub variables of hub $\mathrm{H}_{1}$, the voltage magnitudes and angles of node 1 , the pressure at node 1 , the compressor settings of both compressors, the active power generation $P_{\mathrm{e}, 1}^{\mathrm{G}}$ and the natural gas production $P_{\mathrm{g}}^{\mathrm{G}}$. The second and third control areas are defined analogously. In particular, we assume that:

- control agent 1 sets $P_{\mathrm{e}, 1}^{\mathrm{G}}, P_{\mathrm{g}}^{\mathrm{G}}$, and $\nu_{\mathrm{g}, 1}$;

- control agent 2 sets $P_{\mathrm{e}, 2}^{\mathrm{G}}$ and $\nu_{\mathrm{g}, 2}$;

- control agent 3 sets $P_{\mathrm{e}, 3}^{\mathrm{G}}$ and $\nu_{\mathrm{g}, 3}$.

The control objectives for the three agents result in

$$
\begin{aligned}
& J_{1}\left(\mathbf{x}_{1}, \mathbf{u}_{1}\right)=q_{1}^{\mathrm{G}}\left(P_{\mathrm{e}, 1}^{\mathrm{G}}\right)^{2}+q^{\mathrm{N}}\left(P_{\mathrm{g}}^{\mathrm{G}}\right)^{2} \\
& J_{2}\left(\mathbf{x}_{2}, \mathbf{u}_{2}\right)=q_{2}^{\mathrm{G}}\left(P_{\mathrm{e}, 2}^{\mathrm{G}}\right)^{2} \\
& J_{3}\left(\mathbf{x}_{3}, \mathbf{u}_{3}\right)=q_{3}^{\mathrm{G}}\left(P_{\mathrm{e}, 3}^{\mathrm{G}}\right)^{2},
\end{aligned}
$$

where $\mathbf{x}_{1}=\left[V_{1}, \theta_{1}, p_{1}, p_{\text {inc }, 1}, p_{\text {inc }, 2}, P_{\mathrm{e}, 1}^{\mathrm{H}}, P_{\mathrm{g}, 1}^{\mathrm{H}}\right]^{\mathrm{T}}$, $\mathbf{u}_{1}=\left[P_{\mathrm{e}, 1}^{\mathrm{G}}, P_{\mathrm{g}}^{\mathrm{G}}, \nu_{\mathrm{g}, 1}\right]^{\mathrm{T}}, \quad \mathbf{x}_{2}=\left[V_{2}, \theta_{2}, p_{2}, P_{\mathrm{e}, 2}^{\mathrm{H}}, P_{\mathrm{g}, 2}^{\mathrm{H}}\right]^{\mathrm{T}}$, $\mathbf{u}_{2}=\left[P_{\mathrm{e}, 2}^{\mathrm{G}}, \nu_{\mathrm{g}, 2}\right]^{\mathrm{T}}, \quad \mathbf{x}_{3}=\left[V_{3}, \theta_{3}, p_{3}, P_{\mathrm{e}, 3}^{\mathrm{H}}, P_{\mathrm{g}, 3}^{\mathrm{H}}\right]^{\mathrm{T}}$, and $\mathbf{u}_{3}=\left[P_{\mathrm{e}, 3}^{\mathrm{G}}, \nu_{\mathrm{g}, 3}\right]^{\mathrm{T}}$.

Since the optimization problems of the control agents depend on one another, the agents have to use coordination in order to make sure that they employ the same values for common variables. Some additional terms have to be included in the main objective in order to receive this coordination. In the following section we discuss the approach we use here to solve this problem.

\section{Solving the distributed optimization problem}

To enable coordination within a multi-area system, different decomposition procedures have been elaborated in the last decades. Here, the decomposition procedure proposed in [5] is applied in order to solve the overall optimization problem in a distributed way. The mathematical procedure to decompose a general optimization problem is illustrated on the interconnected two-area system, as depicted in Fig. 3. Extending the procedure to three or more areas is straightforward. The control areas $\mathrm{A}$ and $\mathrm{B}$ comprise the system variables $\left(\mathbf{x}_{\mathrm{A}}, \mathbf{u}_{\mathrm{A}}\right)$ and $\left(\mathbf{x}_{\mathrm{B}}, \mathbf{u}_{\mathrm{B}}\right)$, respectively. Here, only equality constraints are considered. Inequality constraints are handled analogously.

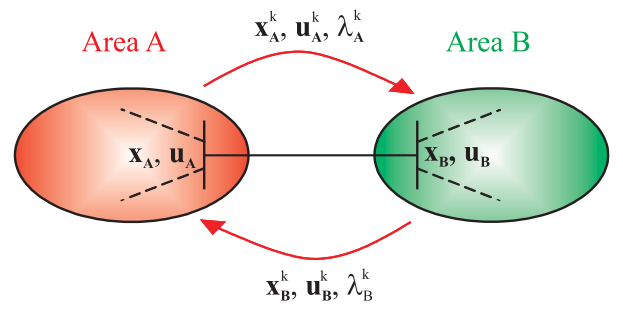

centralized

\begin{tabular}{|l|l|}
$\min _{\mathbf{u}_{\mathrm{A}}, \mathbf{u}_{\mathrm{B}}} J\left(\mathbf{x}_{\mathrm{A}}, \mathbf{u}_{\mathrm{A}}, \mathbf{x}_{\mathrm{B}}, \mathbf{u}_{\mathrm{B}}\right)$ \\
subject to \\
\\
$\mathbf{g}\left(\mathbf{x}_{\mathrm{A}}, \mathbf{u}_{\mathrm{A}}, \mathbf{x}_{\mathrm{B}}, \mathbf{u}_{\mathrm{B}}\right)=0$
\end{tabular}

distributed

\begin{tabular}{|ll}
$\min _{\mathbf{u}_{\mathrm{A}}}$ & $J_{\mathbf{A}}\left(\mathbf{x}_{\mathrm{A}}, \mathbf{u}_{\mathrm{A}}, \mathbf{x}_{\mathrm{B}}^{\mathrm{k}}, \mathbf{u}_{\mathrm{B}}^{\mathrm{k}}\right)$ \\
& $+\left(\lambda_{\mathrm{B}}^{\mathrm{k}}\right)^{\mathrm{T}} \widetilde{\mathrm{g}}_{\mathrm{B}}\left(\mathbf{x}_{\mathrm{A}}, \mathbf{u}_{\mathrm{A}}, \mathbf{x}_{\mathrm{B}}^{\mathrm{k}}, \mathbf{u}_{\mathrm{B}}^{\mathrm{k}}\right)$ \\
subject to & \\
& $\mathbf{g}_{\mathrm{A}}\left(\mathbf{x}_{\mathrm{A}}, \mathbf{u}_{\mathrm{A}}\right)=0$ \\
& $\widetilde{\mathbf{g}}_{\mathrm{A}}\left(\mathbf{x}_{\mathrm{A}}, \mathbf{u}_{\mathrm{A}}, \mathbf{x}_{\mathrm{B}}^{\mathrm{k}}, \mathbf{u}_{\mathrm{B}}^{\mathrm{k}}\right)=0$ \\
\hline
\end{tabular}

distributed

Fig. 3. Decomposition procedure applied to a two-area system. Coupling constraints enable coordination between areas.

For decomposing the centralized optimization problem, the objective and the equality constraints are separated and assigned to the control agent responsible for the component or bus to which the objective and equality constraints are related. There are constraints involving variables of only one area, such as $\mathbf{g}_{\mathrm{A}}\left(\mathbf{x}_{\mathrm{A}}, \mathbf{u}_{\mathrm{A}}\right)$. Besides these constraints, there are also so-called coupling constraints, which are constraints involving variables of both areas, such as $\tilde{g}_{\mathrm{A}}\left(\mathbf{x}_{\mathrm{A}}, \mathbf{u}_{\mathrm{A}}, \mathbf{x}_{\mathrm{B}}, \mathbf{u}_{\mathrm{B}}\right)$, where the tilde is used to indicate coupling constraints. These coupling constraints make that coordination among the control agents of the areas is necessary.

A modified Lagrange relaxation procedure [5] is applied for augmenting the main objective with coupling constraints. As indicated in Fig. 3, the coupling constraints are once added as soft constraints to the main objective of one control agent and once kept explicitly as hard constraints in the constraint set of the control agent controlling the other area. Hence, the objective functions of both control agents consists of two parts. The first term expresses the main objective of each control agent. The second term expresses and enables the coordination. The weighting factors in the second term of the objective function are the Lagrange multipliers obtained from the control agent of the other area.

The optimization problems of the control agents are solved in an iterative way. At each iteration $k$, the optimization problem of each control agent is solved independently of the optimization problem of the other control agent, by keeping the variables of the neighboring area constant. After each iteration, the control agents exchange the updated values of their variables, i.e., the variables $\mathbf{x}_{i}$ and $\mathbf{u}_{i}$ and the Lagrange multipliers $\boldsymbol{\lambda}_{i}$. The iterations stop when the values of the variables exchanged no longer change significantly between two consecutive iterations, viz. the infinity norm of the difference of the values of variables over two iterations 


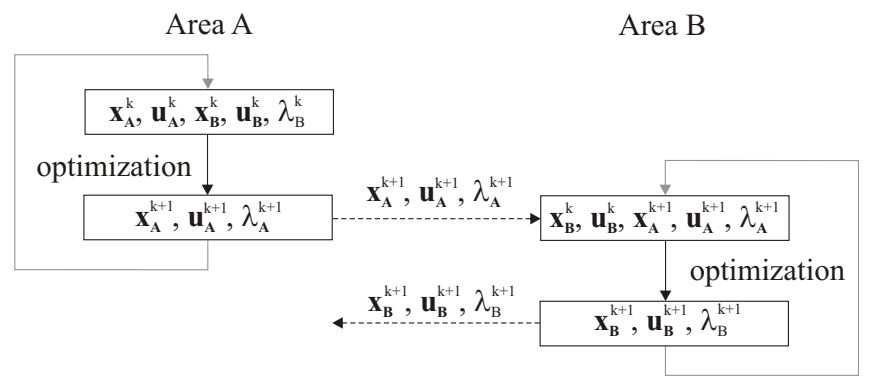

Fig. 4. Information exchange: Serial execution.

is less than a small positive tolerance $\gamma_{\text {tol }}$. As the weighting factors directly result from the neighboring optimization, a fast convergence of the algorithm is obtained when compared to conventional Lagrange relaxation.

Applying this procedure to electric and gas power systems, the power flow equations at the peripheral buses serve as coupling constraints. For the studied three-hub system, the active power balances of all nodes of the electricity system enforce a coordination as they depend on the neighboring voltage magnitudes and angles. For the gas system, the nodal flow balances of all buses enforce the coordination. The injected volume flows are dependent on the nodal pressures of the neighboring buses. Summarizing, for each area, there exists one coupling constraint for the electricity and one for the natural gas system.

\section{Serial versus parallel implementation}

The optimal power flow problem for the entire system is solved using the procedure outlined above. Thereby, each control agent solves the optimization problem for the area it is responsible for. These optimization problems are solved independently, at different locations and by different control entities. In order to coordinate their actions, the control agents have to exchange some information. This information consists of the state and control variables and the Lagrange multipliers. After each completed optimization, the most recent values of these variables are sent to the control agents of neighboring areas. Depending on the application, this information exchange can either be carried out in a serial or in a parallel way.

Fig. 4 illustrates the serial execution of the approach. The control agents solve their optimization problems one after another using the most up-to-date starting values for their variables. For example, the control agent of area A performs its optimization at iteration step $k$ with the values $\mathbf{x}_{\mathrm{A}}^{k}, \mathbf{u}_{\mathrm{A}}^{k}, \mathbf{x}_{\mathrm{B}}^{k}$, $\mathbf{u}_{\mathrm{B}}^{k}, \boldsymbol{\lambda}_{\mathrm{B}}^{k}$. Updated system values $\mathbf{x}_{\mathrm{A}}^{k+1}, \mathbf{u}_{\mathrm{A}}^{k+1}$ and Lagrange multipliers $\lambda_{\mathrm{A}}^{k+1}$ are obtained and sent to the control agent of area B (indicated by the dashed arrows). Now, the control agent of area $\mathrm{B}$ performs its optimization with values $\mathrm{x}_{\mathrm{B}}^{k}$, $\mathbf{u}_{\mathrm{B}}^{k}, \mathbf{x}_{\mathrm{A}}^{k+1}, \mathbf{u}_{\mathrm{A}}^{k+1}, \boldsymbol{\lambda}_{\mathrm{A}}^{k+1}$. Having completed its optimization, the control agent of area $\mathrm{B}$ sends the updated variables $\mathrm{x}_{\mathrm{B}}^{k+1}$, $\mathbf{u}_{\mathrm{B}}^{k+1}, \lambda_{\mathrm{B}}^{k+1}$ back to the control agent of area $\mathrm{A}$, which can proceed with the next iteration $k+1$. The light arrows indicate the optimization process for each area.

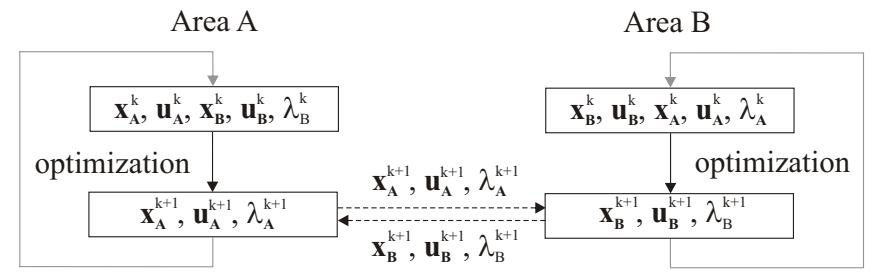

Fig. 5. Information exchange: Parallel execution.

Fig. 5 illustrates the parallel execution of the approach. The control agents solve their optimization problem within each iteration simultaneously, at the same time, starting from equal values at the beginning of the iterations. As soon as both control agents have determined the new values for their variables, they exchange the values of these variables. When all updated values are available to both control agents, the next iteration step is started.

Generally, a serial implementation requires a fewer number of iterations than a parallel implementation to obtain convergence. However, a serial implementation typically requires more time before reaching convergence than a parallel implementation, as is illustrated in [7].

\section{Simulations}

Simulations are made to compare the serial and parallel coordination scheme and properties with respect to convergence speed are discussed. The values of the distributed approach are in each case compared with the centralized optimization which serves as a reference of optimality. The solver fmincon provided by the Optimization Toolbox of Matlab is used [11]. Simulation results are presented, applying the serial and the parallel implementation to the three-hub benchmark system depicted in Fig. 1. The coefficients and simulation parameters used can be found in [4].

The overall objective function to be minimized is defined as in (12) with $q_{1}^{\mathrm{G}}=2, q_{2}^{\mathrm{G}}=4, q_{3}^{\mathrm{G}}=4$, and $q^{\mathrm{N}}=0.2$. In Table I, the control variables obtained by centralized optimization are given yielding a total overall production cost of $\mathrm{TC}=74.118$ p.u. Generator $\mathrm{G}_{1}$ increases its production because its production cost is lower compared with the other two generators. Considering the dispatch factors, the gas turbine in $\mathrm{H}_{1}$ is fully utilized since it is directly connected to the gas network $\mathrm{N}$. Regarding hubs $\mathrm{H}_{2}$ and $\mathrm{H}_{3}$, less gas is converted by the gas turbine as the gas power flows involve compressor losses. The different usages result from the different line losses of the interconnecting pipe lines. The values obtained by the presented distributed coordination method, for both the serial and the parallel execution, deviate within a range of $10^{-3}$ compared to the results applying centralized control and are therefore not explicitly listed.

The values of the active power and gas production over the iterations for both coordination methods are shown in Fig. 6. Applying the parallel approach (Fig. 6(b)), the variables oscillate more until they reach their final values. Considering the objective values of both procedures (solid lines in Fig. 7, denoted by $J_{\text {tot }}$ ) the same behavior is observed. Firstly, 
TABLE I

Simulation RESUlts FOR THREE HUB SYSTEM, CENTRALIZED CONTROL

\begin{tabular}{|l||l|l|l|}
\hline $\mathrm{TC}=74.118$ p.u. & Area 1 & Area 2 & Area 3 \\
\hline \hline$P_{\mathrm{e}, i}^{\mathrm{G}}$ & 3.11 & 1.56 & 1.56 \\
\hline$P_{\mathrm{g}}^{\mathrm{G}}$ & 13.29 & - & - \\
\hline$\nu_{\mathrm{g}, i}$ & 1 & 0.84 & 0.19 \\
\hline
\end{tabular}

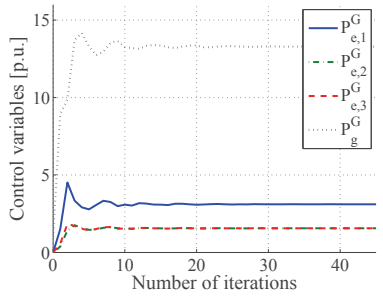

(a)

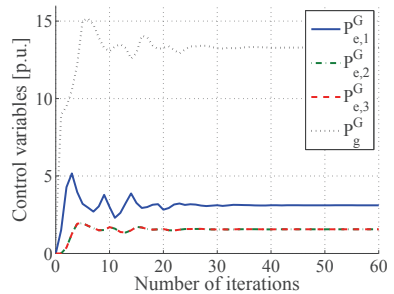

(b)
Fig. 6. Active power generation and natural gas import: (a) serial coordination scheme (b) parallel coordination scheme.

longer oscillations are obtained that in addition have a wider range due to more distinctive coordination problems.

Convergence is achieved when the system variables and the exchanged Lagrange multipliers do not change significantly anymore $\left(\gamma_{\text {tol }}=10^{-3}\right)$. The serial approach (46 iteration steps) yields a faster convergence between the areas than the parallel one (61 iteration steps). As the individual agents start their optimization with the most recent values of the surrounding system, coordination is achieved more easily. For both the serial and the parallel procedure, the slow convergence is amongst others caused by the compressor variables.

To evaluate the coordination between the agents, the coupling constraints are analyzed. In Fig. $7, J_{\text {tot }}$ refers to the total overall objective value and $J$ to the generation costs according to (12). The second term of the objective causing the coordination is denoted by $J_{\text {coupl }}$. As explicated above, $J_{\text {coupl }}$ consists of the three active power balances of the electricity system and the three nodal flow balances of the gas system. At each iteration step it holds $J_{\text {tot }}=J+J_{\text {coupl }}$. As can be noticed, the value of the total objective approaches the generation costs with decaying coupling constraints. With increasing iterations, the coupling constraints decrease to zero, i.e. are fulfilled, indicating that a coordination between the control agents has been achieved. For the parallel scheme (Fig. 7(b)), the coupling constraints take higher absolute values compared with the serial approach, which again illustrates the more pronounced coordination difficulties.

\section{CONClusions And Future Research}

In this paper we have discussed issues in distributed control of combined electricity and natural gas networks. In particular, communication and cooperation issues of a recently proposed control scheme have been addressed. We have elaborated on the differences in performance between

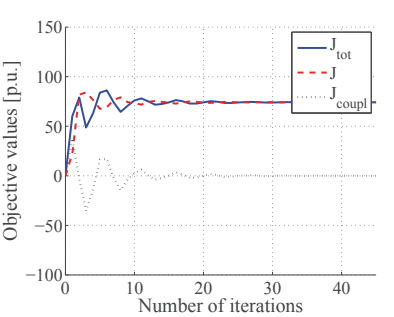

(a)

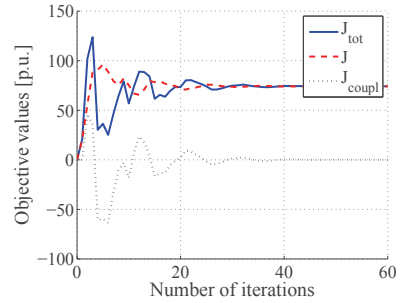

(b)
Fig. 7. Progression of overall objective value $J_{\text {tot, }}$ generation costs $J$ and coupling constraints $J_{\text {coupl applying (a) serial approach (b) parallel }}$ approach.

a serial and parallel implementation of the scheme. Simulations on a three-hub network have illustrated our findings.

Future research should address the extension of relevant dynamics (e.g., of generators and loads), the inclusion of additional control objectives (e.g., on voltage magnitudes and line loadings), and the inclusion of storage devices. In addition, it would be interesting to consider the control of the electricity and gas network being done separately from the control of the energy hubs. In this respect, also issues arising from non-cooperative control agents can be investigated.

\section{ACKNOWLEDGMENTS}

Research supported by the project "Vision of Future Energy Networks" (VoFEN) of ABB, AREVA T\&D, Siemens, and the Swiss Federal Office of Energy, the BSIK project "Next Generation Infrastructures (NGI)", the project "Multi-agent control of large-scale hybrid systems" (DWV.6188) of the Dutch Technology Foundation STW, the European 6th Framework Network of Excellence "HYbrid CONtrol: Taming Heterogeneity and Complexity of Networked Embedded Systems (HYCON)", the European STREP project "Hierarchical and distributed model predictive control (HD-MPC)", and the Delft Research Center Next Generation Infrastructures.

\section{REFERENCES}

[1] K. Hemmes, "Towards multi-source multi-product energy systems," in Proceedings of the 2nd European European Hydrogen Energy Conference (EHEC), Zaragoza, Spain, Nov. 2005.

[2] M. Geidl and G. Andersson, "Optimal power flow of multiple energy carriers," IEEE Transactions on Power Systems, vol. 22, no. 1, pp. 145-155, 2007.

[3] J. Hernandez-Santoyo and A. Sanchez-Cifuentes, "Trigeneration: An alternative for energy savings," Applied Energy, vol. 76, no. 1-3, pp. 219-277, 2003.

[4] M. Arnold and G. Andersson, "Decomposed electricity and natural gas optimal power flow," 16th Power Systems Computation Conference (PSCC 08), 2008.

[5] F. J. Nogales, F. J. Prieto, and A. J. Conejo, "A decomposition methodology applied to the multi-area optimal power flow problem," Annals of Operations Research, vol. 120, pp. 99-116, Apr. 2003.

[6] D. D. Šiljak, Decentralized Control of Complex Systems. Boston, Massachusetts: Academic Press, 1991.

[7] R. R. Negenborn, B. De Schutter, and J. Hellendoorn, "Multi-agent model predictive control for transportation networks: Serial versus parallel schemes," Engineering Applications of Artificial Intelligence, vol. 21, no. 3, pp. 353-366, Apr. 2008.

[8] P. Kundur, Power System Stability and Control. New York, New York: McGraw-Hill, 1994.

[9] E. S. Menon, Gas Pipeline Hydraulics, New York: Taylor \& Francis, 2005.

[10] D. P. Bertsekas, Nonlinear Programming. Beltmore, Massachusetts: Athena Scientific, 2003.

[11] The Mathworks, "Optimization Toolbox User's Guide," 2008. [Online]. Available: http://www.mathworks.com/ 